\title{
A Study on the Relationship between Multicultural Awareness and Multicultural Efficacy in Elementary School Students
}

\author{
Young-Tae $\mathrm{Kim}^{1}$ and Ki-Sung Jang ${ }^{2^{*}}$ \\ ${ }^{1}$ Dept. of Education, Wonkwang University \\ ${ }^{2}$ Dept. of Multicultural Welfare, Wonkwang Health Science University \\ 초등학생의 다문화인식과 다문화효능감과의 관계 연구 \\ 김영태 ${ }^{1}$, 장기성 $2^{*}$ \\ ${ }^{1}$ 원광대학교 교육학과, ${ }^{2}$ 원광보건대학교 다문화복지과
}

\begin{abstract}
The purposes of the study are to understand how multicultural awareness and multicultural efficacy is different and to investigate the factors of multicural awareness affecting the multicultural efficacy. To achieve the goals, the study conducted surveys to examine an interrelation of multicural awareness and efficacy on 329 elementary school students in North Jeolla Province with questionnaires. First, the results showed significant differences in the multicultural awareness and efficacy based on all the ground factors. Second, it was found that elementary school students had significant relations in multicultural awareness and efficacy. Third, the multicultural awareness among elementary school students significantly affected their multicultural efficacy. The study suggests that sustaining multicultural education shall be implemented for elementary school students to have right and positive minds and various studies shall be conducted to nurture values in their lives to live together.
\end{abstract}

요 약 이 연구의 목적은 초등학생의 다문화인식과 다문화효능감은 배경변인별로 어떠한 차이와 상관관계가 있는지 및 다문화인식이 다문화효능감에 영향을 미치는 요인은 무엇인지를 알아보고자 하였다. 이러한 연구목적을 달성하기 위하여 전라북도 초등학교 학생 329 명을 대상으로 질문지를 사용하여 실시하였다. 응답결과에 대하여 첫째, 배경변인 별 모두 다문화인식, 다문화효능감에서 의미있는 차이를 보였다. 둘째, 초등학생들은 다문화인식, 다문화효능감에서 유의미한 상관관계가 있는 것으로 나타났다. 셋째, 초등학생의 다문화인식이 다문화효능감에 유의미한 영향을 미치는 것으로 나타났다. 본 연구결과로 볼 때, 초등학생들에게 다문화에 대한 올바르고 긍정적인 인식을 갖도록 지속적인 다문화교육이 이루어져야 하며, 모두가 더불어 살기 위한 삶의 철학을 기르는 다양한 연구가 진행되어야 할 것으로 사료된다.

Key Words : Multicultural Awareness, Multicultural Efficacy

\section{Introduction}

Recently, the number of foreign labors, marriage migrants, North Korean defectors and foreigners who stay in Korea for a long time has increased, causing to rapidly raise the number of multicultural children[1]. Also, Korea has changed from a single ethic country to a multicultural society with various ethnicities and cultures from various countries and regions[2]. In this sense, it is considered that multicultural families have sharply increased based on this social trend.

Here, multicultural families have faced challenges of

This paper was supported by Wonkwang Health Science University in 2011.

*Corresponding Author : Jang, Ki-Sung(jangkis@wu.ac.kr)

Received January 4, 2012 Revised February 2, 2012

Accepted February 10, 2012 
adapting themselves to a new society[3]. In particular, a unique Korean sociocultural background acts as a factor making the marriage migrants harder for adaptation[4]. This change has rapidly spread to classrooms[5] and here, the multicultural education is defined as educational efforts to make Korean citizens adapt to social changes and live together[2]. Also, the multicultural education can be defined as changing educational institutions including schools and universities to give educational equality to adolescents with different racial, ethnic and social groups[24].

Therefore, families, schools and societies should recognize that children enter the multicultural society, understand more about diversity and grow up with respectful attitudes beyond differences. Children from multicultural families are not strangers to our society. Rather, they are important social members and partners for the future. Children's attitudes toward them are a crucial driving force to integrate our society[6] and multicultural education has been focused from infancy $[1,7]$.

Consciousness of elementary school students toward multicultural education directly affects multicultural efficacy. Therefore, education which gives the students right and positive mindset toward the multicultural society should have priorities through right minds toward multiculture and expanding exchange with the families to nurture the efficacy[8].

To this end, it is required to comprehend multicultural awareness and efficacy among elementary school students, analyze what they need, provide desirable multicultural education and abundant experiences and utilize basic data for real multicultural education. Therefore, the study seeks to provide directions of multicultural education through correlated researches between multicultural awareness and efficacy among elementary school students.

The study topics based on the purposes above are as follows:

$<$ Topic 1> How background factors among elementary school students (gender, grade, achievement, awareness about multicultural families) affect multicultural awareness and efficacy?

$<$ Topic 2> How are multicultural awareness and efficacy among elementary school students related?
$<$ Topic 3> How does the multicultural awareness among elementary school students affect multicultural efficacy?

\section{Method}

\subsection{Subject and Data Collection}

The study was performed for 346 elementary school students in North Jeolla Province. The data were collected from September 5 to 30 in 2011. The respondents were $5^{\text {th }}$ and $6^{\text {th }}$ graders, cooperation from teachers were asked, contents and instructions were informed and let students fill out the questionnaires. A total of 329 questionnaires except 17 with insufficient or poor answers were used for analysis.

Table 1 shows gender, grade, achievement and awareness distributions among the subjects.

[Table 1] General trend of the subject

\begin{tabular}{c|c|c|c}
\hline \multicolumn{2}{c|}{ Spec. } & Respondents & Percentage \\
\hline \hline \multirow{2}{*}{ Gender } & Male & 175 & 53.2 \\
\cline { 2 - 4 } & Female & 154 & 46.8 \\
\hline \multirow{2}{*}{ Level } & 5 th & 162 & 49.2 \\
\cline { 2 - 4 } & 6 th & 167 & 50.8 \\
\hline \multirow{3}{*}{\begin{tabular}{c} 
Achievement \\
\cline { 2 - 4 }
\end{tabular}} & High & 95 & 28.9 \\
\cline { 2 - 4 } & Mid & 171 & 52.0 \\
\hline \multirow{2}{*}{$\begin{array}{c}\text { Recognizing } \\
\text { multicultural } \\
\text { families }\end{array}$} & Low & 63 & 19.1 \\
\cline { 2 - 4 } & Mes & 99 & 30.1 \\
\cline { 2 - 4 } & No & 199 & 60.5 \\
\hline \multicolumn{2}{c}{ All } & 31 & 9.4 \\
\hline \multicolumn{2}{|c}{} & 329 & 100 \\
\hline
\end{tabular}

\subsection{Instruments}

\subsubsection{Multicultural Awareness}

The multicultural awareness means to recognize different cultures from various groups in a society and understand and respect different cultures based on differences what we recognize[9]. The study used questionnaires customized[10] for elder elementary school students developed by[9]. There were 18 questions which consisted of 10 for openness, 4 for acceptance and 4 for respect. The 5-point Likert scale was used and it showed the higher score, the more respectful, open and acceptable to various cultures. The study of[9] showed $.52 \sim .82$ of Cronbach's $a$ and the reliability, openness, acceptance and 
respect of the study showed $.80, .82, .57$ and .51 , respectively.

\subsubsection{Multicultural Efficacy}

The efficacy means confidence and faith required to organize and execute a series of measures to calculate certain results in a situation[8]. The study used questionnaires[12,13] which developed Multicultural Efficacy Scale (MES)[11] and was customized for adolescents. To meet the purpose of the study, the questionnaire was adjusted and complemented for elementary school students requested to 2 elementary school teachers and 2 doctors in the field of education.

There were 12 questions in the questionnaire and consisted of 4 questions for general functions, 4 classes-related questions, 3 for improving social relations and 3 for considering multicultural families. The 5-point Likert scale was used and higher score meant high multicultural efficacy. The study[13] showed $.63 \sim .70$ of Cronbach's $a$ and the reliability, general, class, social relations and multicultural families of Cronbach's a showed $.89, .70, .71, .80$ and .66 , respectively.

\subsection{Data Analysis}

The frequency analysis was performed to understand general features of the subjects and the $\mathrm{F}$ verification was performed by calculating mean and standard deviation to understand differences between multicultural awareness and efficacy from background factors (gender, grade, achievement, awareness). Pearson's product- moment correlation coefficients were calculated to understand sub-factor relations between the multicultural awareness and efficacy and the multiple regression analysis was performed to check how much multicultural awareness affected the multicultural efficacy.

\section{Result and Analysis}

\subsection{Differences between Multicultural Awareness and Efficacy based on Background Factors}

\subsubsection{Differences between Multicultural Awareness and Efficacy based on Gender}

Table 2 shows whether multicultural awareness and efficacy were affected by gender among elementary school students. First, for multicultural awareness, female students showed higher multicultural awareness than that of male counterpart( $\mathrm{F}=22.147, \mathrm{p}<.001)$. Also, the female respondents showed higher scores in sub-factors including openness $(\mathrm{p}<.001)$, acceptance and $\operatorname{respect}(\mathrm{p}<.05)$ for the awareness. Second, the female students showed higher multicultural efficacy than male students $(F=11.106$, $\mathrm{p}<.01$ ). In addition, there were significant differences between male and female students in factors like general $(\mathrm{p}<.05)$, classes, social relations and consideration for multicultural families $(\mathrm{p}<.01)$.

[Table 2] Difference in gender

\begin{tabular}{|c|c|c|c|c|c|c|c|}
\hline \multicolumn{2}{|c|}{ Spec. } & Gender & $\mathrm{N}$ & $\mathrm{M}$ & SD & $\mathrm{F}$ & $\mathrm{p}$ \\
\hline \multirow{12}{*}{$\begin{array}{c}\text { Multicultural } \\
\text { awareness }\end{array}$} & \multirow{3}{*}{ Openness } & Male & 175 & 3.08 & .68 & \multirow{3}{*}{21.771} & \multirow{3}{*}{.000} \\
\hline & & Female & 154 & 3.43 & .69 & & \\
\hline & & All & 329 & 3.24 & .70 & & \\
\hline & \multirow{3}{*}{ Acceptance } & Male & 175 & 3.36 & .81 & \multirow{3}{*}{5.785} & \multirow{3}{*}{.017} \\
\hline & & Female & 154 & 3.57 & .81 & & \\
\hline & & All & 329 & 3.46 & .82 & & \\
\hline & \multirow{3}{*}{ Respect } & Male & 175 & 3.17 & .78 & \multirow{3}{*}{5.104} & \multirow{3}{*}{.025} \\
\hline & & Female & 154 & 3.37 & .78 & & \\
\hline & & All & 329 & 3.26 & .79 & & \\
\hline & \multirow{3}{*}{ All } & Male & 175 & 3.16 & .53 & \multirow{3}{*}{22.147} & \multirow{3}{*}{.000} \\
\hline & & Female & 154 & 3.45 & .57 & & \\
\hline & & All & 329 & 3.29 & .57 & & \\
\hline \multirow{15}{*}{$\begin{array}{c}\text { Multicultural } \\
\text { efficacy }\end{array}$} & \multirow{3}{*}{ General } & Male & 175 & 3.26 & .77 & \multirow{3}{*}{4.966} & \multirow{3}{*}{.027} \\
\hline & & Female & 154 & 3.44 & .70 & & \\
\hline & & All & 329 & 3.34 & .74 & & \\
\hline & \multirow{3}{*}{ Class } & Male & 175 & 3.12 & .81 & \multirow{3}{*}{8.859} & \multirow{3}{*}{.003} \\
\hline & & Female & 154 & 3.37 & .69 & & \\
\hline & & All & 329 & 3.24 & .77 & & \\
\hline & \multirow{3}{*}{$\begin{array}{l}\text { Social } \\
\text { relations }\end{array}$} & Male & 175 & 3.34 & .98 & \multirow{3}{*}{8.146} & \multirow{3}{*}{.005} \\
\hline & & Female & 154 & 3.62 & .82 & & \\
\hline & & All & 329 & 3.47 & .92 & & \\
\hline & \multirow{3}{*}{$\begin{array}{c}\text { Considering } \\
\text { multicultural } \\
\text { families }\end{array}$} & Male & 175 & 3.12 & .89 & \multirow{3}{*}{10.015} & \multirow{3}{*}{.002} \\
\hline & & Female & 154 & 3.40 & .68 & & \\
\hline & & All & 329 & 3.25 & .81 & & \\
\hline & \multirow{3}{*}{ All } & Male & 175 & 3.21 & .72 & \multirow{3}{*}{11.106} & \multirow{3}{*}{.001} \\
\hline & & Female & 154 & 3.45 & .59 & & \\
\hline & & All & 329 & 3.32 & .67 & & \\
\hline
\end{tabular}

\subsubsection{Differences between Multicultural Awareness} and Efficacy based on Grade

Table 3 shows whether multicultural awareness and efficacy were affected by grades among elementary school students. First, sixth graders showed higher multicultural awareness than that of $5^{\text {th }} \operatorname{graders}(\mathrm{F}=18.167, \mathrm{p}<.01)$. Also, the higher grade students showed higher openness $(\mathrm{p}<.001)$. 
A Study on the Relationship between Multicultural Awareness and Multicultural Efficacy in Elementary School Sudents

However, there were no significant differences in acceptance and respect. Second, $6^{\text {th }}$ grade students showed higher multicultural efficacy than the $5^{\text {th }}$ grade students $(\mathrm{F}=4.598, \mathrm{p}<.05)$. The higher grade students showed significant differences in general $(\mathrm{p}<.01)$ and social relations $(\mathrm{p}<.05)$ but no significant differences in classes and social relations.

[Table 3] Difference in level

\begin{tabular}{|c|c|c|c|c|c|c|c|}
\hline \multicolumn{2}{|c|}{ Spec. } & Level & $\mathrm{N}$ & M & SD & $\mathrm{F}$ & $\mathrm{p}$ \\
\hline \multirow{12}{*}{$\begin{array}{c}\text { Multicultural } \\
\text { awareness }\end{array}$} & \multirow{3}{*}{ Openness } & $\overline{5^{\text {th }}}$ & 162 & 3.01 & ב.70 & \multirow{3}{*}{39.767} & \multirow{3}{*}{.000} \\
\hline & & $6^{\text {th }}$ & 167 & 3.47 & .63 & & \\
\hline & & All & 329 & 3.24 & .70 & & \\
\hline & \multirow{3}{*}{ Acceptance } & $5^{\text {th }}$ & 162 & 3.42 & .73 & \multirow{3}{*}{.753} & \multirow{3}{*}{.386} \\
\hline & & $6^{\text {th }}$ & 167 & 3.50 & .89 & & \\
\hline & & All & 329 & 3.46 & .82 & & \\
\hline & \multirow{3}{*}{ Respect } & $5^{\text {th }}$ & 162 & 3.29 & .72 & \multirow{3}{*}{.519} & \multirow{3}{*}{.472} \\
\hline & & $6^{\text {th }}$ & 167 & 3.23 & .85 & & \\
\hline & & All & 329 & 3.26 & .79 & & \\
\hline & \multirow{3}{*}{ All } & $5^{\text {th }}$ & 162 & 3.16 & .53 & \multirow{3}{*}{18.167} & \multirow{3}{*}{.000} \\
\hline & & $6^{\text {th }}$ & 167 & 3.42 & .57 & & \\
\hline & & All & 329 & 3.29 & .57 & & \\
\hline \multirow{15}{*}{$\begin{array}{c}\text { Multicultural } \\
\text { efficacy }\end{array}$} & \multirow{3}{*}{ General } & $5^{\text {th }}$ & 162 & 3.22 & .72 & \multirow{3}{*}{8.879} & \multirow{3}{*}{.003} \\
\hline & & $6^{\text {th }}$ & 167 & 3.46 & .75 & & \\
\hline & & All & 329 & 3.34 & .74 & & \\
\hline & \multirow{3}{*}{ Class } & $5^{\text {th }}$ & 162 & 3.20 & .77 & \multirow{3}{*}{.647} & \multirow{3}{*}{.422} \\
\hline & & $6^{\text {th }}$ & 167 & 3.27 & .77 & & \\
\hline & & All & 329 & 3.24 & .77 & & \\
\hline & \multirow{3}{*}{$\begin{array}{l}\text { Social } \\
\text { relations }\end{array}$} & $5^{\text {th }}$ & 162 & 3.37 & .93 & \multirow{3}{*}{4.179} & \multirow{3}{*}{.042} \\
\hline & & $6^{\text {th }}$ & 167 & 3.57 & .89 & & \\
\hline & & All & 329 & 3.47 & .92 & & \\
\hline & \multirow{3}{*}{$\begin{array}{l}\text { Considering } \\
\text { multicultural } \\
\text { families }\end{array}$} & $5^{\text {th }}$ & 162 & 3.19 & .78 & \multirow{3}{*}{1.843} & \multirow{3}{*}{.176} \\
\hline & & $6^{\text {th }}$ & 167 & 3.31 & .83 & & \\
\hline & & All & 329 & 3.25 & .81 & & \\
\hline & \multirow{3}{*}{ All } & $5^{\text {th }}$ & 162 & 3.24 & .66 & \multirow{3}{*}{4.598} & \multirow{3}{*}{.033} \\
\hline & & $6^{\text {th }}$ & 167 & 3.40 & .68 & & \\
\hline & & All & 329 & 3.32 & .67 & & \\
\hline
\end{tabular}

\subsubsection{Differences between Multicultural Awareness} and Efficacy based on Achievement

Table 4 shows whether multicultural awareness and efficacy were affected by achievement among elementary school students. First, students with higher achievement showed higher multicultural awareness than that of their counterpart $(\mathrm{F}=5.930, \quad \mathrm{p}<.01)$. Only openness among sub-factors $(\mathrm{p}<.01)$ showed higher multicultural awareness. Second, students with higher achievement showed higher multicultural efficacy than their counterpart $(\mathrm{F}=19.956$, $\mathrm{p}<.001)$. Among sub-factors, general, class and social relations $(\mathrm{p}<.001)$ and consideration $(\mathrm{p}<.01)$ showed higher trend among students with higher achievement.

[Table 4] Difference in achievement

\begin{tabular}{|c|c|c|c|c|c|c|c|c|}
\hline \multicolumn{2}{|c|}{ Spec. } & $\begin{array}{c}\text { Achieve } \\
\text { ment }\end{array}$ & $\mathrm{N}$ & M & $\mathrm{SD}$ & $\mathrm{F}$ & $\mathrm{p}$ & Scheffe \\
\hline \multirow{16}{*}{$\begin{array}{c}\text { Multicultural } \\
\text { awareness }\end{array}$} & \multirow{4}{*}{ Openness } & $\operatorname{High}(1)$ & 95 & 3.29 & .69 & \multirow{4}{*}{5.669} & \multirow{4}{*}{.004} & \multirow{4}{*}{$1,2>3$} \\
\hline & & $\operatorname{Mid}(2)$ & 171 & 3.31 & .66 & & & \\
\hline & & Low(3) & 63 & 2.98 & .77 & & & \\
\hline & & All & 329 & 3.24 & .70 & & & \\
\hline & \multirow{4}{*}{ Acceptance } & High & 95 & 3.50 & .84 & \multirow{4}{*}{2.025} & \multirow{4}{*}{.134} & \\
\hline & & Mid & 171 & 3.51 & .80 & & & \\
\hline & & Low & 63 & 3.27 & .81 & & & \\
\hline & & All & 329 & 3.46 & .82 & & & \\
\hline & \multirow{4}{*}{ Respect } & High & 95 & 3.28 & .84 & \multirow{4}{*}{1.184} & \multirow{4}{*}{.307} & \\
\hline & & Mid & 171 & 3.30 & .76 & & & \\
\hline & & Low & 63 & 3.13 & .77 & & & \\
\hline & & All & 329 & 3.26 & .79 & & & \\
\hline & \multirow{4}{*}{ All } & $\operatorname{High}(1)$ & 95 & 3.34 & .61 & \multirow{4}{*}{5.930} & \multirow{4}{*}{.003} & \multirow{4}{*}{$1,2>3$} \\
\hline & & $\operatorname{Mid}(2)$ & 171 & 3.35 & .54 & & & \\
\hline & & Low(3) & 63 & 3.08 & .53 & & & \\
\hline & & All & 329 & 3.29 & .57 & & & \\
\hline \multirow{20}{*}{$\begin{array}{c}\text { Multicultural } \\
\text { efficacy }\end{array}$} & \multirow{4}{*}{ General } & $\operatorname{High}(1)$ & 95 & 3.63 & .72 & \multirow{4}{*}{22.876} & \multirow{4}{*}{.000} & \multirow{4}{*}{$1>2>3$} \\
\hline & & $\operatorname{Mid}(2)$ & 171 & 3.36 & .69 & & & \\
\hline & & Low(3) & 63 & 2.86 & .68 & & & \\
\hline & & All & 329 & 3.34 & .74 & & & \\
\hline & \multirow{4}{*}{ Class } & High(1) & 95 & 3.44 & .81 & \multirow{4}{*}{15.456} & \multirow{4}{*}{.000} & \multirow{4}{*}{$1,2>3$} \\
\hline & & $\operatorname{Mid}(2)$ & 171 & 3.29 & .65 & & & \\
\hline & & Low(3) & 63 & 2.79 & .83 & & & \\
\hline & & All & 329 & 3.24 & .77 & & & \\
\hline & \multirow{4}{*}{$\begin{array}{c}\text { Social } \\
\text { relations }\end{array}$} & $\operatorname{High}(1)$ & 95 & 3.68 & .96 & & & \\
\hline & & $\operatorname{Mid}(2)$ & 171 & 3.56 & .80 & 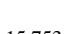 & & 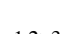 \\
\hline & & Low(3) & 63 & 2.92 & .93 & $15 . / 53$ & .000 & $1,2>3$ \\
\hline & & All & 329 & 3.47 & .92 & & & \\
\hline & & $\operatorname{High}(1)$ & 95 & 3.20 & .79 & & & \\
\hline & Considering & $\operatorname{Mid}(2)$ & 171 & 3.39 & .69 & & & \\
\hline & (1) & Low(3) & 63 & 2.94 & 1.04 & & & \\
\hline & & All & 329 & 3.25 & .81 & & & \\
\hline & & $\operatorname{High}(1)$ & 95 & 3.50 & .70 & & & \\
\hline & 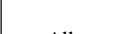 & $\operatorname{Mid}(2)$ & 171 & 3.39 & .57 & & & \\
\hline & All & Low(3) & 63 & 2.87 & .71 & 19.950 & .000 & \\
\hline & & All & 329 & 3.32 & .67 & & & \\
\hline
\end{tabular}

\subsubsection{Differences between Multicultural Awareness} and Efficacy based on Recognition

Table 5 shows whether multicultural awareness and efficacy were affected by recognizing multicultural families among elementary school students. First, it was shown that students who 'knew' multicultural families showed higher recognition than those who 'did not know' 
multicultural awareness( $\mathrm{F}=10.687, \quad \mathrm{p}<.001)$. Among sub-factors, students who 'knew' multicultural families showed higher awareness than those who 'did not know' the families among openness $(\mathrm{p}<.001)$ and acceptance $(\mathrm{p}<.05)$. Second, students who 'knew' multicultural families showed higher multicultural efficacy than those who 'did not know' it $(\mathrm{F}=25.833, \mathrm{p}<.001)$. The respondents who 'knew' the concept showed higher efficacy than those who 'did not know' among all the sub-factors $(\mathrm{p}<.001)$.

[Table 5] Difference in recognizing multicultural families

\begin{tabular}{|c|c|c|c|c|c|c|c|c|}
\hline \multicolumn{2}{|c|}{ Spec. } & $\begin{array}{c}\text { Recogniz } \\
\text { ing }\end{array}$ & $\mathrm{N}$ & M & SD & $\mathrm{F}$ & $\mathrm{p}$ & Scheffe \\
\hline \multirow{16}{*}{$\begin{array}{c}\text { Multicultural } \\
\text { awareness }\end{array}$} & \multirow{4}{*}{ Openness } & Yes(1) & 99 & 3.49 & .69 & \multirow{4}{*}{10.260} & \multirow{4}{*}{.000} & \multirow{4}{*}{$1>2,3$} \\
\hline & & Maybe(2) & 199 & 3.16 & .68 & & & \\
\hline & & $\mathrm{No}(3)$ & 31 & 2.96 & .68 & & & \\
\hline & & All & 329 & 3.24 & .70 & & & \\
\hline & \multirow{4}{*}{ Acceptance } & Yes & 99 & 3.66 & .83 & \multirow{4}{*}{4.696} & \multirow{4}{*}{.010} & \\
\hline & & Maybe & 199 & 3.36 & .82 & & & \\
\hline & & No & 31 & 3.45 & .62 & & & \\
\hline & & All & 329 & 3.46 & .82 & & & \\
\hline & \multirow{4}{*}{ Respect } & Yes & 99 & 3.40 & .85 & \multirow{4}{*}{2.148} & \multirow{4}{*}{.118} & \\
\hline & & Maybe & 199 & 3.20 & .78 & & & \\
\hline & & No & 31 & 3.23 & .49 & & & \\
\hline & & All & 329 & 3.26 & .79 & & & \\
\hline & \multirow{4}{*}{ All } & Yes(1) & 99 & 3.51 & .60 & \multirow{4}{*}{10.687} & \multirow{4}{*}{.000} & \multirow{4}{*}{$1>2,3$} \\
\hline & & Maybe(2) & 199 & 3.22 & .54 & & & \\
\hline & & $\mathrm{No}(3)$ & 31 & 3.13 & .42 & & & \\
\hline & & All & 329 & 3.29 & .57 & & & \\
\hline \multirow{20}{*}{$\begin{array}{c}\text { Multicultural } \\
\text { efficacy }\end{array}$} & \multirow{4}{*}{ General } & Yes(1) & 99 & 3.70 & .66 & \multirow{4}{*}{19.638} & \multirow{4}{*}{.000} & \multirow{4}{*}{$1>2,3$} \\
\hline & & Maybe(2) & 199 & 3.23 & .74 & & & \\
\hline & & $\mathrm{No}(3)$ & 31 & 2.95 & .59 & & & \\
\hline & & All & 329 & 3.34 & .74 & & & \\
\hline & \multirow{4}{*}{ Class } & Yes(1) & 99 & 3.62 & .66 & \multirow{4}{*}{21.102} & \multirow{4}{*}{.000} & \multirow{4}{*}{$1>2,3$} \\
\hline & & Maybe(2) & 199 & 3.11 & .71 & & & \\
\hline & & $\mathrm{No}(3)$ & 31 & 2.85 & .95 & & & \\
\hline & & All & 329 & 3.24 & .77 & & & \\
\hline & \multirow{4}{*}{$\begin{array}{c}\text { Social } \\
\text { relations }\end{array}$} & Yes(1) & 99 & 3.83 & .81 & & & \\
\hline & & Maybe(2) & 199 & 3.39 & .86 & & & \\
\hline & & $\mathrm{No}(3)$ & 31 & 2.86 & 1.11 & & & \\
\hline & & All & 329 & 3.47 & .92 & & & \\
\hline & & Yes(1) & 99 & 3.57 & .77 & & & \\
\hline & Considering & Maybe(2) & 199 & 3.14 & .75 & & & \\
\hline & families & $\mathrm{No}(3)$ & 31 & 2.93 & 1.04 & & & \\
\hline & & All & 329 & 3.25 & .81 & & & \\
\hline & & Yes(1) & 99 & 3.68 & .57 & & & \\
\hline & & Maybe(2) & 199 & 3.21 & .63 & & & \\
\hline & & $\mathrm{No}(3)$ & 31 & 2.90 & .78 & & & \\
\hline & & All & 329 & 3.32 & .67 & & & \\
\hline
\end{tabular}

\subsection{Verifying Correlation}

Table 6 shows the results of the correlation analysis to discover relations between multicultural awareness and efficacy. The results showed that there were significant relations between sub-factors like openness, acceptance and respect of the awareness and general, class, social relations and consideration for multicultural families, the sub-factors $(\mathrm{p}<.01)$. Based on this, each factor of multicultural awareness and efficacy has relations.

[Table 6] Correlations for each factor

\begin{tabular}{|c|c|c|c|c|c|c|c|}
\hline \multirow{2}{*}{ Spec. } & \multicolumn{3}{|c|}{ Multicultural awareness } & \multicolumn{4}{|c|}{ Multicultural efficacy } \\
\hline & (1) & (2) & (3) & (4) & (5) & (6) & (7) \\
\hline (2) & $.266^{(* *)}$ & & & & & & \\
\hline (3) & $.177^{(* *)}$ & $.715^{(* *)}$ & & & & & \\
\hline (4) & $.520^{(* *)}$ & $.233^{(* *)}$ & $.147^{(* *)}$ & & & & \\
\hline (5) & $.385^{(* \star)}$ & $.222^{(* \star)}$ & $.205^{(* *)}$ & $.642^{(* *)}$ & & & \\
\hline (6) & $.433^{(* *)}$ & $.259^{(* *)}$ & $.208^{(* *)}$ & $.603^{(* *)}$ & $.706^{(* *)}$ & & \\
\hline (7) & $.448^{(* *)}$ & $.237^{(* *)}$ & $.199^{(* *)}$ & $.490^{(* *)}$ & $.588^{(\star *)}$ & $.591^{(* *)}$ & \\
\hline
\end{tabular}

(5) Class (6) Social relations (7) Considering multicultural families

\subsection{How Multicultural Awareness Affects the Efficacy}

Table 7 shows how the multicultural awareness affects sub-factors of efficacy including general, class, social relations and consideration for multicultural families. The stepwise regression analysis including significantly related variables was performed to understand factors in multicultural awareness.

As a result of analyzing multi-collinearity among independent variables, the tolerance range was between .469 and .929 , and the Variation Inflation Factor(VIF) was between 1.077 and 2.132, no problem in the multi-collinearity.

The results of multiple regression analysis showed general features were affected by openness $(\mathrm{t}=10.112$, $\mathrm{p}<.001)$ and acceptance $(\mathrm{t}=2.076, \mathrm{p}<.05)$ and these factors affected $27.6 \%$ of the general. Classes were affected by openness $(\mathrm{t}=7.018, \mathrm{p}<.001)$ and $\operatorname{respect}(\mathrm{t}=2.743, \mathrm{p}<.01)$, having $16.2 \%$ of effect. Social relations were affected by openness $(\mathrm{t}=7.664, \mathrm{p}<.001)$ and acceptance $(\mathrm{t}=3.027, \mathrm{p}<.01)$, affecting $20.5 \%$ of the relations. Consideration was affected by openness $(\mathrm{t}=8.419, \mathrm{p}<.001)$ and acceptance 
$(\mathrm{t}=2.483, \mathrm{p}<.05)$, accounting for $21.1 \%$ of the relations.

[Table 7] Factors in multicultural awareness affecting multicultural efficacy

\begin{tabular}{|c|c|c|c|c|c|c|}
\hline Spec. & model & B & $\mathrm{SE}$ & $\beta$ & $\mathrm{t}$ & $\mathrm{p}$ \\
\hline \multirow{4}{*}{ General } & (Constant) & 1.333 & .198 & & 6.724 & .000 \\
\hline & Openness & .522 & .052 & .493 & 10.112 & .000 \\
\hline & Acceptance & .092 & .044 & .101 & 2.076 & .039 \\
\hline & \multicolumn{6}{|c|}{ Adj R2=.276 $\quad F=63.379 \quad \mathrm{p}<.001$} \\
\hline \multirow{4}{*}{ Class } & (Constant) & 1.518 & .226 & & 6.724 & .000 \\
\hline & Openness & .393 & .056 & .360 & 7.018 & .000 \\
\hline & Respect & .138 & .050 & .141 & 2.743 & .006 \\
\hline & \multicolumn{6}{|c|}{ Adj $\mathrm{R} 2=.162 \quad \mathrm{~F}=32.818 \quad \mathrm{p}<.001$} \\
\hline \multirow{4}{*}{$\begin{array}{l}\text { Social } \\
\text { relations }\end{array}$} & (Constant) & 1.225 & .255 & & 4.803 & .000 \\
\hline & Openness & .509 & .066 & .392 & 7.664 & .000 \\
\hline & Acceptance & .173 & .057 & .155 & 3.027 & .003 \\
\hline & \multicolumn{6}{|c|}{ Adj $R 2=.205 \quad F=43.198$} \\
\hline \multirow{4}{*}{$\begin{array}{l}\text { Considering } \\
\text { multicultural } \\
\text { families }\end{array}$} & (Constant) & 1.272 & .225 & & 5.653 & .000 \\
\hline & Openness & .478 & .059 & .415 & 8.419 & .000 \\
\hline & Acceptance & .125 & .051 & .126 & 2.483 & .014 \\
\hline & \multicolumn{6}{|c|}{ Adj $R 2=.211 \quad F=44.868$} \\
\hline
\end{tabular}

\section{Conclusion and Discussion}

The study aimed at how multicultural awareness and efficacy were different, related and the awareness affected the efficacy. The results and discussion in the study are as follows:

First, the awareness and the efficacy showed significant differences in the gender. The sub-factors in the multicultural awareness showed higher among female students than their counterpart. Also, the sub-factors in the efficacy showed significant differences.

Second, the awareness and the efficacy showed significant differences in the level. $6^{\text {th }}$ graders showed higher openness than the $5^{\text {th }}$ graders, as well as general and social relations in the efficacy. However, there were no differences in classes and social relations.

Third, the awareness and the efficacy showed significant differences in the achievement. Students with higher achievement showed higher openness than their counterpart in the multicultural awareness. However, the sub-factors of the multicultural efficacy showed high trend.
Fourth, the awareness and the efficacy showed significant differences in recognizing multicultural families. Here, those who 'knew' multicultural families showed higher awareness than those who 'did not know'. Also, the former showed higher awareness of sub-factors in the efficacy than the latter.

Fifth, significant static relations existed in the multicultural awareness and efficacy. This showed that openness, acceptance and respect of the awareness and general, class, social relations and consideration for multicultural families of the efficacy had relations with each other.

Sixth, the multiple regression analysis of factors how multicultural awareness affected the efficacy showed all the sub-factors in the awareness had a significant effect $(\mathrm{p}<.001)$. Here, openness and acceptance affected general features of $27.6 \%$. Openness and respect affected classes of $16.2 \%$. Openness and acceptance affected social relations of $21.6 \%$. Openness and acceptance affected the consideration of $21.1 \%$.

The result showed that the multicultural awareness and efficacy among elementary school students showed a significant difference in background factors. Especially, female students showed higher awareness and efficacy than their male counterpart. This supports that the female students had higher awareness than the male students[14-17] and it suggests that concentrated and distinctive education for male students are required[8]. Also, students who knew multicultural families showed higher awareness and efficacy than their counterpart. This supports studies showing that students with educational background of multicultural education [5,15,16, 18-22], it is very important to have an education focusing on various cultures[8] and it is clear to analyze how much children understand multicultural awareness, what they need and provide proper education and abundant experience [17]. In particular, it is required to provide knowledge and experience for elementary school students to have positive minds and naturally view the society to enhance multicultural efficacy in the multicultural society.

Also, it was found that the multicultural awareness directly affected the efficacy. Therefore, there shall be specific education to understand and respect each culture and have positive minds toward other cultures to enhance multicultural efficacy. Especially, elementary school 
students are naturally exposed to multicultural environment. It is considered that multicultural education is important to have positive and open minds toward people with different races and ethnicity beyond distinguishing the majority and the minority[23]. Also, it suggests that teachers and parents shall take aggressive attitudes in schools and families to help students have more positive and active minds toward multi-culture and multicultural efficacy.

The limitations in the study and suggestions for following studies are as follows:

First, the subjects of the study were elementary school students in North Jeolla Province. Therefore, the study had limitation on generalizing the results to all the elementary school students and following studies shall expand the subjects and include elementary and middle school students in other regions.

Second, questionnaires were used to investigate multicultural awareness and efficacy and there was no method to investigate in-depth contents. It means the study may not be applied to understanding specific and various contents.

Third, there shall be studies which develop and apply reasonable and specific multicultural education programs for elementary school students to teach philosophy for living together

\section{References}

[1] Shim, W. Y., Elementary School Students' Attitudes toward Multicultural Children, The Journal of Elementary Education, 23(4), pp. 43 63, 2010.

[2] Chang, I. S., Multicultural Education in America and Curriculum. The Journal of Curriculum Studies, 24(4), pp. 27 53, 2006.

[3] Hong, D, A, G,. \& Chae, O. H., \& Lee, N. J., Development and Evaluation of Family Integration Education Programs for Multi-Cultural Families, Korean Journal of Human Ecology , 15(5), pp. 729 741, 2006.

[4] Do, M. H., \& Kim, S. C., A Study for Perception Increase of Childcare Teacher on Multicultural Family Education. Korean Journal of Family Welfare, 15(3), pp. 163 181, 2010.

[5] Kim, M. H., Influence of Program Based on Bennett's Multicultural Education Model upon Multicultural
Awareness and Self-esteem. Graduate School of Education, Gyeongin National University of Education, Master's Thesis, 2009.

[6] Lawson, K. M., Komar, B., \& Rose, A. M.(1998). Building one nation : a study of what is being done today in schools, neighborhoods and the workplace. Washington, DC : Leadership Conference Education Fund.

[7] Lee, K. Y., \& Choi, S. Y,. \& Jang, S. W., \& Shin, S. C., Recognition of Early Childhood Teachers and Parents about Multicultural Education, The Korean Journal Child Education, 19(1), pp. 115 132, 2010.

[8] Cho, Y. J., The Effects of Perceptions of Multiculture on Efficiency Esteem of Multiculture in Elementary School Students, Journal of Korean Practical Arts Education, 23(3), pp. 43 58, 2010.

[9] Jeong, G. M., "Influence of Anti-Bias Educational Program on Bias and Multicultural Awareness". Graduate School of Education, Gyeongin National University of Education, Master's Thesis, 2009.

[10] Rothfarb, Sylvia H., Perception of intergroup relations in secondary schools of Miami Dade County Public Schools : Results of the student multicultural relations survey, Office of Educational Accountability, Miami-Dade County Public Schools, 1992.

[11] Guyton, E. M \& Wesche, M. V., "The Multicultural Efficacy Scale: Development, Item Selection, and Reliability", Multicultural Perspective, 7(4), pp. 21 29, 2005.

[12] Choi, C. O., \& Mo, K. H., Investigating Multicultural Efficacy of Teachers in Gyeonggi Province, Theory and Research in Citizenship Education, 39(4), pp. 163 182, 2007.

[13] Lim, J. Y., Perception and Self Efficiency Esteem of the Multiculture Among the Adolescents. Graduate School of Education, Kyunghee University, Master's Thesis, 2009.

[14] Kim, A. Y., The Research of Elementary School Teacher's Recohnition and Zctual Condition regarding Multicultural Education, Graduate School of Education, Seoul National University of Education, Master's Thesis, 2006

[15] Park, J. W., How elementary school students understand "the international marriage"?, Graduate School of Education, Korea National University of Education, Master's Thesis, 2007.

[16] O, J. S., A Study on Recognition of Elementary Students about Multi-cultural Family Children. Graduate 
School, Cheongju University, Master's Thesis, 2009.

[17] Lee, H. J., A Study on Factors to Influence Multicultural Awareness of Elementary School Students. Graduate School of Education, Gyeongin National University of Education, Master's Thesis, 2009.

[18] Lim, G. Y., The Effects of Multicultural Education using Children's Books in Elementary Social Studies. Graduate School of Education, Chongju National University of Education, Master's Thesis, 2008.

[19] Lim, G. Y., A Study of Effectiveness of Multi-cultural Education Program. Graduate School of , Sogang University, Master's Thesis, 2008.

[20] Lee, K. Y., The Effects of the Multi-cultural Education Activities on Young Children's Anti-bias Recognition and Attitudes toward Other Cultures, The Korean Journal Child Education, 17(2), pp. 65 79, 2008.

[21] Choi, A. B., The Effects of the Integrated Multicultural Education Program on Children's Recognition and Attitudes toward Cultural Aspects. Graduate School, Kookmin University, Ph.D Thesis, 2009.

[22] Yoo, Y. S., The Influence of Multicultural Educational Program on Multicultural Awareness and Self-Identity. Graduate School of Education, Gyeongin National University of Education, Master's Thesis, 2009.

[23] Yang, Y. J., Directions of Curriculum Development of Multicultural Education in Korean Society. Graduate School, Ewha Womans University, Ph.D Thesis, 2008.

[24] Banks, J. A., Cultural diversity and education: Foundation, curriculum, and teaching. Boston: Pearson Education, Inc. 2006.

\section{Young-Tae Kim}

[Regular member]

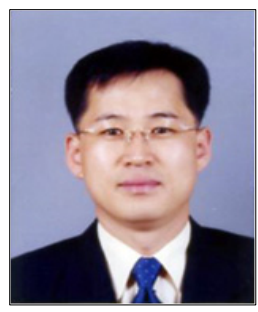

- Feb. 1991 : Wonkwang Univ. Dept. of education

- Feb. 1995 : Wonkwang Univ. Dept. of education, ME

- Feb. 2007 : Wonkwang Univ. Dept. of education, $\mathrm{PhD}$
Ki-Sung Jang

[Regular member]

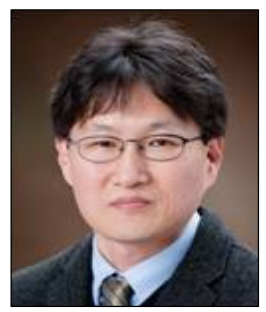

- Feb, 1987 : Wonkwang Univ. Dept. of English Education

- Feb, 1989 : Wonkwang Univ. Dept. English Literature \& Language, ME

- Feb. 1996 : Wonkwang Univ. Dept. English Literature \& Language, $\mathrm{PhD}$

- Sep. 2009 current : Professor, Dept of Multicultural Welfare, Wonkwang Helath Science Univ.

$<$ Research Interests $>$

Multicultural Education, Korean Education for Foreigner

$<$ Research Interests $>$

Educational Psychology, Multicultural Education, Curriculum \& Educational Evaluation, 